\title{
Fatou type weighted pointwise convergence of nonlinear singular integral operators Depending on two parameters
}

\author{
Gumrah Uysal $^{1}$ and Sevilay Kirci Serenbay ${ }^{2}$ \\ ${ }^{1}$ Karabuk University, Faculty of Science, Department of Mathematics, Karabuk, Turkey \\ ${ }^{2}$ Baskent University, Faculty of Education, Department of Mathematic Education, Ankara, Turkey
}

Abstract. In this paper we present some theorems concerning existence and Fatou type weighted pointwise convergence of nonlinear singular integral operators of the form:

$$
\left(T_{\lambda} f\right)(x)=\int_{\mathrm{R}} K_{\lambda}(t-x ; f(t)) d t, x \in \mathrm{R}, \lambda \in \Lambda
$$

where $\Lambda \neq \varnothing$ is a set of non-negative indices, at a common generalized Lebesgue point of the functions $f \in L_{1, \phi}(\mathrm{R})$ and positive weight function $\phi$. Here, $L_{1, \phi}(\mathrm{R})$ is the space of all measurable functions for which $\left|\frac{f}{\phi}\right|$ is integrable on $\mathrm{R}$.

\section{Introduction}

In [1], Taberski analyzed the pointwise approximation to functions $f \in L_{1}\langle-\pi, \pi\rangle$ and their derivatives by a family of convolution type linear singular integral operators depending on two parameters of the form:

$L_{\lambda}(f ; x)=\int_{-\pi}^{\pi} f(t) K_{\lambda}(t-x) d t, x \in\langle-\pi, \pi\rangle, \lambda \in \Omega \subset \mathrm{R}_{0}^{+}$

where the symbol $\langle-\pi, \pi\rangle$ stands for closed, semiclosed or open interval and $K_{\lambda}(t)$ is the kernel satisfying suitable assumptions. Subsequently, the pointwise convergence of the operators of above type was examined by Gadjiev [2] and Rydzewska [3] at generalized Lebesgue points and $\mu$ - generalized Lebesgue points of function $f \in L_{1}\langle-\pi, \pi\rangle$ respectively. Besides, Mamedov [4] and Taberski [5] obtained significant results on weighted pointwise convergence of linear singular integral operators. For some recent studies on singular integrals, authors refer to [6-8].

Later on, Musielak [9, 10] improved the notion of singularity to include the case of nonlinear integral operators of the form:

$$
\left(T_{w} f\right)(y)=\int_{G} K_{w}(x-y ; f(x)) d x, y \in G, w \in \Lambda
$$

where $G$ be a locally compact Abelian group equipped with Haar measure and $\Lambda \neq \varnothing$ be an index set with any topology, via replacing the linearity property of the operators by an assumption of Lipschitz condition for $K_{w}$ with respect to second variable. The studies, which were published until that time, showed that the singularity of the operators was related to their linearity [11]. Afterwards, Swiderski and Wachnicki [12] investigated the pointwise convergence of the operators of the preceding type at Lebesgue points of the functions $f \in L_{p}(-\pi, \pi)$.

Nowadays, approximation via nonlinear integral operators is extensively used in many branches of science such as medicine and engineering. Especially, effect of nonlinear integral operators in sampling theory must be emphasized here [11]. Further, signal and image processing are two major research fields around sampling theory. In view of this situation, one may choose to study the convergence of nonlinear integral operators rather than the convergence of linear integral operators. For further studies concerning Fatou type convergence of the singular integral operators, authors refer to [13-15].

The current manuscript deals with the Fatou type weighted pointwise convergence of nonlinear singular integral operators of the form:

$$
\left(T_{\lambda} f\right)(x)=\int_{\mathrm{R}} K_{\lambda}(t-x ; f(t)) d t, x \in \mathrm{R}, \lambda \in \Lambda
$$

where $\Lambda \neq \varnothing$ be the set of indices, at generalized Lebesgue point of the functions $f \in L_{1, \phi}(\mathrm{R})$ and $\phi$. Here, $L_{1, \varphi}(\mathrm{R})$ is the space of all measurable functions for which $\left|\frac{f}{\phi}\right|$ is integrable on $\mathrm{R}$.

In Section 2, we introduce fundamental notions. In Section 3, we prove the existence of the operators. In 
Section 4, we give a theorem concerning the pointwise convergence of $\left(T_{\lambda} f\right)(x)$.

\section{Preliminaries}

Following [12, 14], let $G$ be the locally compact Abelian group equipped with Haar measure. In addition, $N(\vartheta)$ stands for the family of all neighborhoods of the identity element $\vartheta \in G$ and $\vartheta_{0}$ is an accumulation point of $G$. Let $\Lambda \neq \varnothing$ be a set of indices with any topology and $\lambda_{0}$ be an accumulation point of this set in this topology.

Definition 1. (Class $\left.A_{\phi}\right)$ Suppose that the weight function $\phi: \mathrm{R} \rightarrow \mathrm{R}^{+}$is bounded on arbitrary bounded subsets of $\mathrm{R}$ and the inequality:

$$
\phi(t+x) \leq \phi(t) \phi(x), \quad t \in \mathrm{R}, x \in \mathrm{R}
$$

holds. We will say that the family of the functions $K_{\lambda}: G \times \mathrm{R} \rightarrow \mathrm{R}$, where $K_{\lambda}(\vartheta, u)$ is Haar integrable on $G$ for all $u \in \mathrm{R}$, belongs to Class $A_{\phi}$ if the following conditions are satisfied:

a) $K_{\lambda}(\vartheta, 0)=0$ for every $\vartheta \in G$ and $\lambda \in \Lambda$.

b) There exists an integrable function $L_{\lambda}: G \rightarrow \mathrm{R}$ such that the following inequality:

$$
\left|K_{\lambda}(t, u)-K_{\lambda}(t, v)\right| \leq L_{\lambda}(t)|u-v|,
$$

holds $\forall t \in G, \forall u, v \in \mathrm{R}$ and for each $\lambda \in \Lambda$.

c) $\forall u \in \mathrm{R}$, we have

$$
\lim _{(x, \lambda) \rightarrow\left(x_{0}, \lambda_{0}\right)}\left|\int_{G} K_{\lambda}\left(t-x ; \frac{u}{\phi\left(x_{0}\right)} \phi(t)\right) d t-u\right|=0,
$$

where $x_{0} \in \mathrm{R}$ is a generalized Lebesgue point of $\phi$.

d)

$$
\lim _{\lambda \rightarrow \lambda_{0}} \sup _{t \in G \backslash \Omega}\left[\phi(t) L_{\lambda}(t)\right]=0, \quad \forall \Omega \in N(\vartheta) .
$$

e)

$$
\lim _{\lambda \rightarrow \lambda_{0}}\left[\int_{G \backslash \Omega} \phi(t) L_{\lambda}(t) d t\right]=0, \quad \forall \Omega \in N(\vartheta) .
$$

f) For a given $\delta_{1}>0, L_{\lambda}(t)$ is non-decreasing function as a function of $\mathrm{t}$ on $\left\langle-\delta_{1}, 0\right]$ and non-increasing function as a function of $\mathrm{t}$ on $\left[0, \delta_{1}\right\rangle$.

$$
\text { g) }\left\|\phi L_{\lambda}\right\|_{L_{1}(G)} \leq M<\infty, \quad \forall \lambda \in \Lambda .
$$

Throughout this article, we assume that $G=\mathrm{R}$ and $K_{\lambda}$ belongs to class $A_{\phi}$.
Definition 2. [16] A generalized Lebesgue point of a locally integrable function $g: \mathrm{R} \rightarrow \mathrm{R}$ is a point $x_{0} \in \mathrm{R}$ satisfying

$$
\lim _{h \rightarrow 0} \frac{1}{h^{\alpha+1}} \int_{x_{0}}^{x_{0}+h}\left|g(t)-g\left(x_{0}\right)\right| d t=0, \quad 0 \leq \alpha \leq N .
$$

Example 1. Let $f: \mathrm{R} \rightarrow \mathrm{R}$ be given by

$$
f(t)= \begin{cases}t e^{-t}, & t \in(0,1] \\ 0, & \text { otherwise }\end{cases}
$$

It is easy to see that $x_{0}=0$ is a generalized Lebesgue point of the given function for $\alpha=\frac{1}{4}$.

\section{Existence of the Operators}

Main result in this work is based on the following theorem.

Theorem 1. If $f \in L_{1, \phi}(\mathrm{R})$, then the operator $\left(T_{\lambda} f\right)(x) \in L_{1, \varphi}(\mathrm{R})$ and

$$
\left\|\left(T_{\lambda} f\right)\right\|_{L_{1, \phi}(\mathrm{R})} \leq\left\|\phi L_{\lambda}\right\|_{L_{1}(\mathrm{R})}\|f\|_{L_{1, \phi}(\mathrm{R})}
$$

for all $\lambda \in \Lambda$.

Proof: Using Fubini's Theorem [17] we may write

$$
\begin{aligned}
\left\|\left(T_{\lambda} f\right)(x)\right\|_{L_{1, \phi}(\mathrm{R})} & =\int_{-\infty}^{\infty} \frac{1}{\phi(x)}\left|\int_{-\infty}^{\infty} K_{\lambda}(t-x ; f(t)) d t\right| d x \\
& \leq \int_{-\infty}^{\infty} \frac{1}{\phi(x)} \int_{-\infty}^{\infty}\left|K_{\lambda}(t-x ; f(t))-K_{\lambda}(t-x ; 0)\right| d t d x \\
& \leq \int_{-\infty}^{\infty} \frac{1}{\phi(x)} \int_{-\infty}^{\infty}\left|f(t+x) \frac{\phi(t+x)}{\phi(t+x)} L_{\lambda}(t)\right| d t d x \\
& \leq \int_{-\infty}^{\infty} L_{\lambda}(t)\left[\int_{-\infty}^{\infty} \frac{\phi(t) \phi(x)}{\phi(x)}\left|\frac{f(t+x)}{\phi(t+x)}\right| d x\right] d t \\
& \leq\left\|\phi L_{\lambda}\right\|_{L_{1}(\mathrm{R})}\|f\|_{L_{1, \phi}(\mathrm{R})},
\end{aligned}
$$

where the norm of $f$ is given by the following equality (see, e.g., [4]):

$$
\|f\|_{L_{1, \phi}(R)}=\int_{-\infty}^{\infty}\left|\frac{f(x)}{\phi(x)}\right| d x .
$$

\section{Convergence at characteristic points}

In this section we prove the Fatou type weighted pointwise convergence of the operators $\left(T_{\lambda} f\right)(x)$, i.e. the convergence will be restricted to the bounded planar subsets of $\mathrm{R} \times \Lambda$. For this purpose, we define the set: 


$$
Z=\left\{(x, \lambda) \in \mathrm{R} \times \Lambda: \Omega_{\delta}(x, \lambda)<C\right\},
$$

where $C$ is any positive constant and for $\delta$ values satisfying $0<\delta<\delta_{1}$, the function $\Omega_{\delta}$ is defined by

$$
\begin{aligned}
& \Omega_{\delta}(x, \lambda)= \\
& \sup _{t \in\left\langle x_{0}-\delta, x_{0}+\delta\right\rangle} \phi(t)\left(\begin{array}{l}
\int_{x_{0}-\delta}^{x_{0}+\delta} L_{\lambda}(t-x)\left|\left\{\left|t-x_{0}\right|^{\alpha+1}\right\}_{t}^{\prime}\right| d t \\
+2 L_{\lambda}(0)\left|x-x_{0}\right|^{\alpha+1}
\end{array}\right),
\end{aligned}
$$$$
0 \leq \alpha \leq N \text {. }
$$

Theorem 1. If $x_{0} \in \mathrm{R}$ is a common generalized Lebesgue point of the functions $f \in L_{1, \phi}(\mathrm{R})$ and $\phi$ then

$$
\lim _{(x, \lambda) \rightarrow\left(x_{0}, \lambda_{0}\right)}\left|\left(T_{\lambda} f\right)(x)-f\left(x_{0}\right)\right|=0
$$

as $(x, \lambda)$ tends to $\left(x_{0}, \lambda_{0}\right)$ whenever $(x, \lambda) \in Z$.

Proof: Let $0<x_{0}-x<\frac{\delta}{2}$ for $\delta$ which satisfies $\delta_{1}>\delta>0$. Set $I=\left|\left(T_{\lambda} f\right)(x)-f\left(x_{0}\right)\right|$. Now, using condition $(c)$ of class $A_{\phi}$, we may write

$$
\begin{aligned}
I= & \left|\int_{-\infty}^{\infty} K_{\lambda}(t-x ; f(t)) d t-f\left(x_{0}\right)\right| \\
= & \mid \int_{-\infty}^{\infty} K_{\lambda}(t-x ; f(t)) d t-\int_{-\infty}^{\infty} K_{\lambda}\left(t-x ; \frac{f\left(x_{0}\right)}{\phi\left(x_{0}\right)} \phi(t)\right) d t \\
& +\int_{-\infty}^{\infty} K_{\lambda}\left(t-x ; \frac{f\left(x_{0}\right)}{\phi\left(x_{0}\right)} \phi(t)\right) d t-f\left(x_{0}\right) \mid .
\end{aligned}
$$

Using condition $(b)$ of class $A_{\phi}$, the following inequality:

$$
\begin{aligned}
I & \leq \int_{-\infty}^{\infty}\left|\frac{f(t)}{\phi(t)}-\frac{f\left(x_{0}\right)}{\phi\left(x_{0}\right)}\right| \phi(t) L_{\lambda}(t-x) d t \\
& +\left|\int_{-\infty}^{\infty} K_{\lambda}\left(t-x ; \frac{f\left(x_{0}\right)}{\phi\left(x_{0}\right)} \phi(t)\right) d t-f\left(x_{0}\right)\right| \\
& =I_{1}+I_{2}
\end{aligned}
$$

is obtained. Using condition (c) of class $A_{\phi}, I_{2} \rightarrow 0$ as $(x, \lambda)$ tends to $\left(x_{0}, \lambda_{0}\right)$. By the hypothesis, it is easy to see that the following inequality for $I_{1}$,

$$
\begin{aligned}
I_{1} & \leq \Omega_{\delta}(x, \lambda)+\int_{\mathrm{R} \backslash\left\langle x_{0}-\delta, x_{0}+\delta\right\rangle}\left|\frac{f(t)}{\phi(t)}-\frac{f\left(x_{0}\right)}{\phi\left(x_{0}\right)}\right| \phi(t) L_{\lambda}(t-x) d t \\
& \leq \delta \Omega_{\delta}(x, \lambda)+\phi(x)\|f\|_{L_{1, \phi}(\mathrm{R})} \sup _{|u| \geq \frac{\delta}{2}}\left[\phi(u) L_{\lambda}(u)\right] \\
& +\phi(x)\left|\frac{f\left(x_{0}\right)}{\phi\left(x_{0}\right)}\right| \int_{|u| \mid \frac{\delta}{2}} \phi(u) L_{\lambda}(u) d u,
\end{aligned}
$$

holds.

Therefore, if the points $(x, \lambda) \in Z$ are sufficiently near to $\left(x_{0}, \lambda_{0}\right)$, then the first term on the right hand side of the above inequality tends to zero.

Also, remaining two terms tend to zero by conditions $(d)$ and $(e)$ of class $A_{\phi}$, respectively. Using similar method, one may obtain the same result for the case $0<x-x_{0}<\frac{\delta}{2}$. Hence, the proof is completed.

\section{Conclusion}

In this paper, the weighted pointwise convergence for the family of nonlinear convolution type singular integral operators depending on two parameters is investigated. This study may be seen as a continuation and generalization of the previous studies, such as $[1,12]$. Since the approximation results and the character of the kernel function are related, a special class of kernel functions, called class $A_{\phi}$, has been defined. Therefore, the main result is presented as Theorem 1 .

\section{References}

1. R. Taberski, Prace Mat. 7, 173-179 (1962)

2. A. D. Gadjiev, The order of convergence of singular integrals which depend on two parameters, in: Special Problems of Functional Analysis and Their Applications to the Theory of Differential Equations and the Theory of Functions, Izdat. Akad. Nauk Azerbaidžan SSR., Baku, 40-44 (1968)

3. B. Rydzewska, Fasc. Math. 7, 71-81 (1973)

4. R. G. Mamedov, Izv. Akad. Nauk. SSSR Ser. Mat. 27 (2), 287-304 (1963)

5. R. Taberski, Comment. Math. Prace Mat. 19 (1), 155-160 (1976)

6. G. Uysal, M. M. Yilmaz, E. Ibikli, J. Inequal. Appl. 2015, 94 (2015)

7. G. Uysal, E. Ibikli, J. Pure Appl. Math. Adv. Appl. 14 (2), 151-166 (2015)

8. G. Uysal, E. Ibikli, New Trends Math. Sci. 4 (1), 104-114 (2016)

9. J. Musielak, On some approximation problems in modular spaces, in: Constructive Function Theory Publ. House Bulgarian Acad. Sci., Sofia , 455-461, (1983)

10. J. Musielak, Comment. Math. Prace Mat., 31, 79-88 (1991)

11. C. Bardaro, J. Musielak, G. Vinti, Nonlinear integral 
operators and applications, DeGruyter Series in Nonlinear Analysis and Applications, 9 (2003)

12. T. Swiderski, E. Wachnicki, Comment. Math. 40, 181-189 (2000)

13. H. Karsli, E. Ibikli, Fasc. Math., 38, 25-39 (2007)

14. M. Carlsson, Math. Scand. 102 (2), 231-252 (2008)

15. H. Karsli, Appl. Math. Comput. 246, 221-228 (2014)

16. R. G. Mamedov, A study of orders of convergence of one-dimensional and multidimensional singular integrals, in: Studies in Theory of Differential Equations and Theory of Functions, Izdat. Akad. Nauk Azerbaĭdžan. SSR, Baku, 92-108 (1965)

17. P. L. Butzer, R. J. Nessel, Fourier Analysis and Approximation, vol. I. Academic Press, New York, London, (1971) 